\title{
Social Skills Group for Adults Living with Asperger's Syndrome
}

\author{
Fabienne Giuliani* and Pierre El Korh \\ Psychiatry and Mental Development Department, Community Psychiatry Service, Psychiatry Department, CHUV, Switzerland
}

Corresponding author: Fabienne Giuliani, Psychiatry and Mental Development Department, Community Psychiatry Service, Psychiatry Department, CHUV, Switzerland, Tel: +41213142185; E-mail: Fabienne.Giuliani@chuv.ch

Rec date: June 09, 2016; Acc date: July 12, 2016; Pub date: July 31, 2016

Copyright: (C) 2016 Giuliani F et al. This is an open-access article distributed under the terms of the Creative Commons Attribution License, which permits unrestricted use, distribution, and reproduction in any medium, provided the original author and source are credited.

Citation: Giuliani F, El Korh P. Social Skills Group for Adults Living with Asperger's Syndrome. Clin Psychiatry. 2016, 2:3.

\section{Abstract}

This work presents the results obtained in a therapeutic social skills group of adults living with Asperger's Syndrome (ASD). The treatment consists in a regular participation in specifically designed groups. Patients meeting the criteria for ASD have been selected with no psychiatric comorbidity and were thus able to optimal group interaction. They were suffering significant anxiety symptoms supposed to lead to inadequate social skills as well as to result from them. Requisite participation included ten sessions in group discussions of topics propose by the patients themselves. Special attention was accord to train the patients in detecting possible functional analysis processes leading to increasing anxiety and in training social skills. The paper concentrates in three assessments by the patient themselves of four different scales (anxiety, depression, self-esteem and social skills in daily life) allowing to the comparison of baseline level (before session 1) with short term (immediately after the last session) and long term benefits of training (3 months later). These different measures revealed significant long term improvement in the patients. These results are important because they consist in training the patients in self-help. They might also contribute to better understanding of the ASD by the scientific community as well as by the patients themselves. Finally, long term treatments such as proposed here are more likely to extend the improvement of the patients' well-being to their social environment, family and professional one. It is thus both a clinical and a theoretically relevant research effort.

Keywords: Peer helping; Social skills; Therapeutic groups; Cognitive behavioral therapy; Psychotherapy; Asperger's syndrome

\section{Introduction}

Autism spectrum disorders (ASD) are neuro-developmental disorders and affect men four times more than women. The prevalence is estimated at 1: 5000 in 1975, 1: 2500 in 1985, 1: 500 in 1995, 1: 110 in 2009, 1:88 in 2012 and today estimated at 1:68 [1,2]. As explained by Hadjikhani [3], autism spectrum disorders are now more frequent than childhood cancers, juvenile diabetes and pediatric AIDS, and pose significant challenges for both public health and education. Individuals living with an ASD experience: 1) altered social communication, 2) restricted interests, repertories and behaviors. Following the publication of the fifth edition of the DSM, Asperger's Syndrome is no longer differentiated as a specific category of autism, it is now included beneath the larger designation of autism spectrum disorders without mental retardation whose symptoms lie on the same continuum [4]. Nevertheless, as our study began prior to the publication of the DSM-V, we will speak of Asperger's Syndrome in this article.

Several studies have established that the greatest problems for Asperger's individuals are anxiety disorders [5-10]. A recent study has reported that in a sample of young adults with Asperger's, approximately $50 \%$ also experienced an anxiety disorder and other studies suggest that individuals with Asperger's are highly susceptible, at some point in their life, of developing serious difficulties through a range of anxiety disorders (for example, panic disorders and generalized anxiety disorder) [11-13]. For this work we hypothesized that anxiety disorders in individuals with Asperger's Syndrome could be caused by a lack of social skills and so we worked to focus our therapy on social skills building and through this hoped to reduce the anxiety disorders.

Cognitive behavioral therapy (CBT) based on social skills has already been shown to be an effective treatment for anxiety in individuals with Asperger's Syndrome and there is increasing interest concerning therapies for people with Asperger's who experience difficulties linked to anxiety [14-19].

Recently, studies have begun to look at aspects of the group therapeutic process for this population $[5,19]$. The analyses provided by these authors have shown, on the one hand, that patients have played an active role in the therapy and, on the other hand, that the context of the therapeutic group will change patient behavior.

In the Lugnegard et al. study [11], anxiety disorders were observed in approximately $50 \%$ of all young adults with Asperger's and this sub-group experienced at least one episode of major depression (70\%) or recurring episodes of depression (50\%). Psychotic disorders and disorders induced 
by substance abuse were rare. The researchers concluded that young adults with autism spectrum disorders were at a higher risk for anxiety disorders and mood disorders. Mental health disorders can arise from a multitude of factors which influence each other (biological, psychological, sociological and/or environmental). Which is why it is necessary to conduct an initial clinical evaluation in order to gather information on the causes of patient difficulties.

Some authors [20-22] have nevertheless underscored the importance of the behavioral evaluation before undertaking an intervention and within the framework of group cognitive behavioral therapy, to evaluate the social skills of patients, and so this is what we have done.

\section{Methods}

\section{Evaluation of interpersonal difficulties}

The group addresses the following types of interpersonal difficulties, either specific or combined:

1) Social anxiety: patients may feel very anxious in a certain number of interpersonal situations

2) Lack of social skills: patients may have difficulty expressing certain needs, ideas or feelings, either in any interpersonal situation or in front of particular individuals;

3) A poor perception of his or her own skills: patients may perceive themselves to be socially inept without this being true;

4) A poor perception of the social-interpersonal reality: patients may erroneously perceive the messages they receive from others and interpret them incorrectly;

5) Difficulty managing one's emotions (name, describe, communicate)

6) A combination of these five factors.

\section{Elements of the evaluation}

The four main points of our initial evaluation of interpersonal difficulties are the following:

1) What difficulties does the patient encounter?

2) In which situations do these difficulties appear? The term "situations" is taken here in the broad sense, including physical and interpersonal aspects, verbal and nonverbal actions and reactions of others. In other words, everything in the patient's environment that can call forth or reinforce the patient's behaviors, emotions and cognitions.

3) What are the behavioral, emotional, and cognitive "chains" as well as what situations lead the patient to manifest problematic behaviors?

4) What are the behaviors, emotions or cognitions that would be better for the patient to learn and manifest in order to resolve these difficulties?

\section{Measurements taken}

We conducted a number of psychopathological evaluations. The following measurements were taken at T0, T1 and T2:

- Beck Anxiety Inventory

- 13-item Beck Depression Inventory

- Rosenberg Self-esteem Scale

- The STAI (French version)

We conducted systematic group measurements in the following way (These measures come from a systematic questionnaire given at the end of each group session):

- State of fatigue before/after the group

- Is that the group is helpful for you? (Usefulness of the group)

- Were the facilitators energetic?

- Were the facilitators flexible in group interactions?

Repeated variance analyses (ANOVA with the Statview 4.5 software) were used in order to compare the results obtained between T0, T1 and T2, at first on the different scales but also on each group participation. Fisher's post-hoc and other simple correlation comparisons were performed.

\section{Practice of social skills groups}

Make-up of the groups: Our patients $(n=30)$ are adults with Asperger's Syndrome ( 8 women and 22 men) between the ages of 20 and 43 years (average of $28.9 \pm 7$ ). These participants didn't know each other before group formation. The Asperger's Syndrome diagnosis of these patients corresponded to the diagnostic criteria of the DSM-IV [23]. Furthermore, the individuals in our group did not have any psychiatric comorbidity. Inclusion criteria were: (1) participants meet the diagnostic criteria for Asperger's Syndrome according to the DSM-IV; (2) participants have clinically significant symptoms of anxiety (Hamilton scale $>4$ ); (3) they have a score between $<41$; $>50$ on the Global Assessment of Functioning scale from the DSM-IV [23]; (4) participants are between the ages of 18 and 65.

Exclusion criteria were: (1) participants suffering from posttraumatic stress syndrome, or disorders linked to substance abuse; (2) serious psychiatric comorbidities which would impair their ability to interact in a group.

The semi-open groups were kept small (between 4 to 6 participants) with ten monthly sessions per year. Each session was recorded and lasted 90 minutes. The study lasted four years. All participants consented to participate in the study.

To begin with, we specified the interpersonal difficulties in the way that we described them previously using the scales we will present hereafter (Table 1). In order to ensure compliance of the therapeutic treatment, we took inspiration from [24] in terms of developing specific goals. The facilitators of the group are the first and second authors. The authors are experienced and both hold a specialist's degree in BCT. 
We developed our own approach inspired by CBT $[25,26]$ and we will soon publish our approach in the form of a therapist's manual meant for Asperger's patients.

Sessions were organized in the following manner:

Sessions began via order of the day, meaning a round table discussion of the problematic subjects that the patient may have experienced and wants to tackle in the group (Figure 1). We noted all subjects on a board and we voted upon which subjects the group wants to work on. The other subjects were listed on a monitoring sheet so there was a record of all subjects brought up as well as the individuals who brought them to the group.

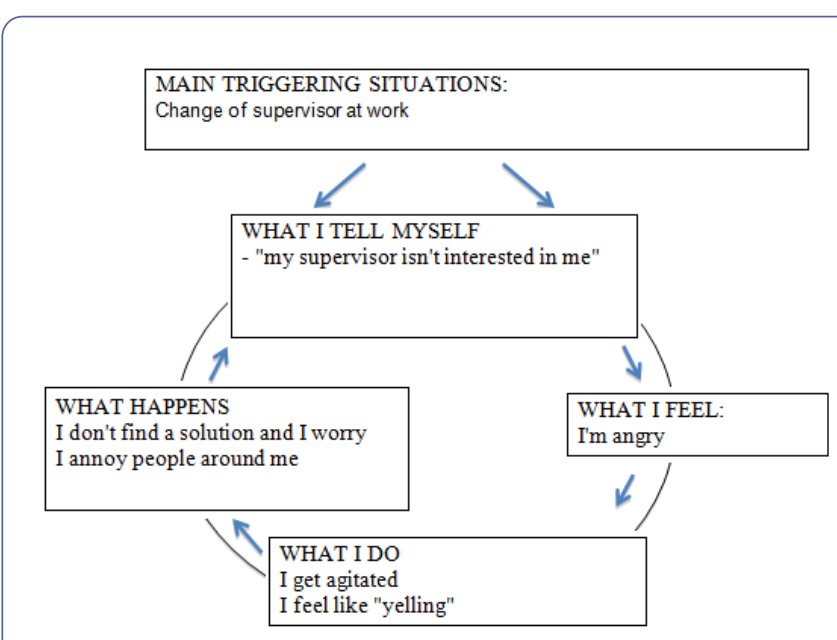

Figure 1 Example of a patient's vicious circle occurring during a group session.

\section{Session program}

Session 1: We covered what it means to participate in this group: the notion of social skills, the notion of self-esteem, the feeling of self-sufficiency, the notion of stressors and the notion of learning via one's peers. We insisted upon the aspects of confidentiality in the group, of written consent for video recording of the sessions and their usefulness.

During this session, we discussed the functional analysis of the situations to be discussed through the vicious circle described by Cungi [27], which enabled us to discuss cognitions, emotions, behaviors and consequences. We were attentive to the interactions between the different group members.

After completing the functional analysis, we went around the table and listed participant experiences of this particular experience. We conducted role plays for this situation. Finally, we finished the session with positive reinforcement.

At the end of the session, we evaluated the session using a questionnaire which specifies participant fatigue on a scale of 0 to 10 before and after the group; the usefulness of the group activity as well as the quality of the animation (energy and flexibility) were evaluated using a Likert scale.
Session 2-10: The following nine sessions occurred in the same manner as the first. We ensured the acquisition of skills in the following: management of emotions, stress, social interactions and cognitions. Additionally, the participants brought us their experience in line with different social scenarios and fundamental needs (Maslow's pyramid). The following techniques were used according to their appropriateness:

Table 1 Social skills.

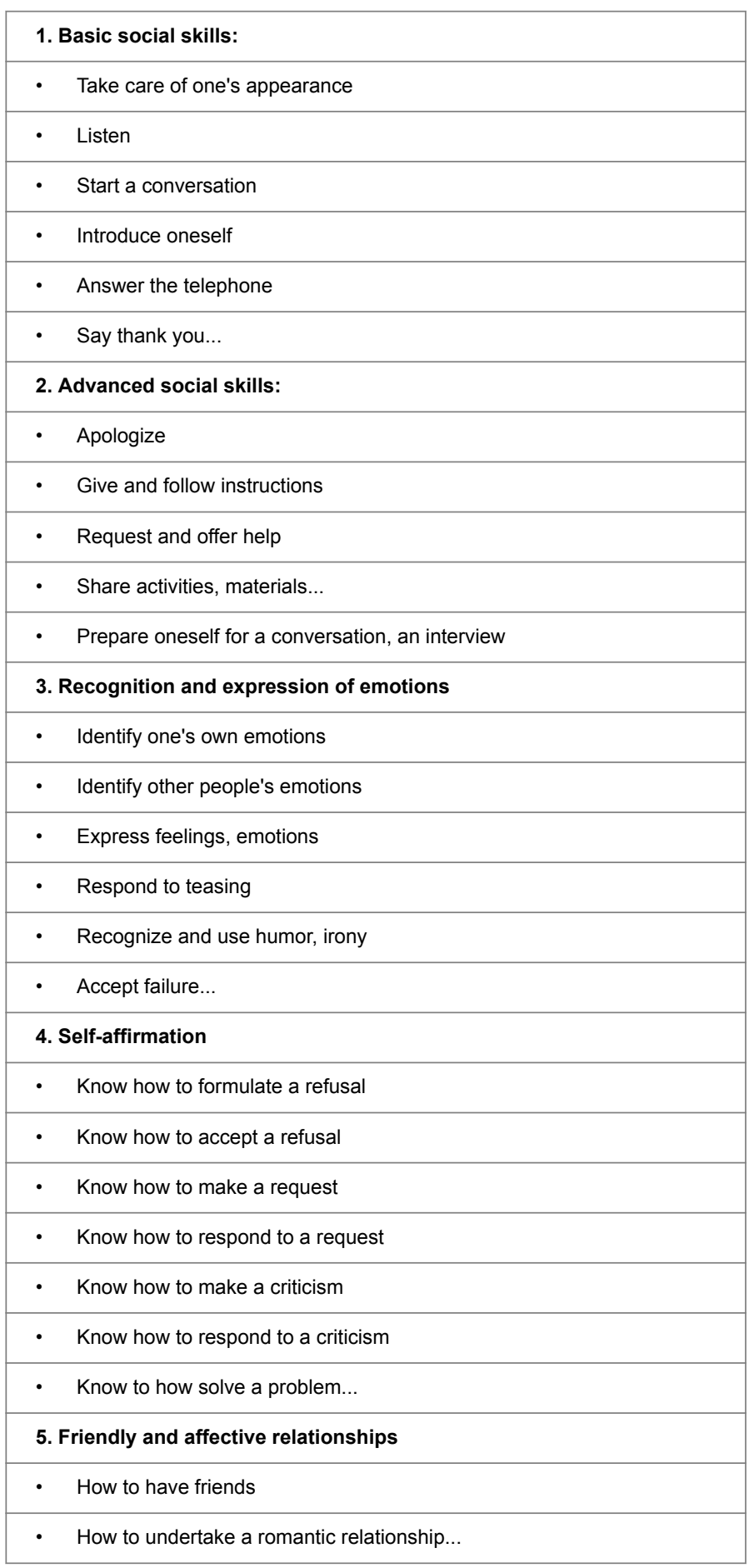

Social skills: Definition: "Listen to what someone has said to me (understand), select a response and reply (through verbal 
or nonverbal means), in an appropriate way for a given social situation."

Rules for self-affirmation, relaxation (through square breathing); formulation of a request or a refusal; formulating or receiving a criticism; managing interpersonal conflicts; working on problem-solving techniques; knowing how to make and receive compliments; knowing how to manage and express negative emotions linked to grieving.

\section{Role playing}

Starting from the situation brought up, role playing games were proposed and undertaken at first by the facilitators and then by the participants. If one of the participants had trouble, we offered help by whispering responses and we repeated the situation until the participant could say it on his or her own. At the end of every role play, we always validated the participant. We used an evaluation table to evaluate participant behaviors on the verbal and nonverbal level. Table $\mathbf{2}$ shows the monitoring sessions. We note: a) the situation brought up; b) which participant has brought the situation; c) role playing games makes by the participant; d) the successful of the role playing games.

\section{Learning via one's peers}

As insisted by Grossen [28], "The therapeutic framework is a collective construction in an external and internal reality," and we were careful to go beyond the classical dichotomy between the patient and his environment by showing that this relationship is always mediated by the interactions of the individual with others.

Table 2 Protocol for role playing session.

\begin{tabular}{|c|c|c|c|c|c|c|c|}
\hline Name & Short term goal + situation & Role playing game & Ok & Not Ok & Summary + Tasks & \multicolumn{2}{|c|}{ Successful } \\
\hline & & Position & & & & Yes & No \\
\hline & & Volume of voice & & & & & \\
\hline & & Tone of voice & & & & & \\
\hline & & Facial Expression & & & & & \\
\hline & & Visual contact & & & & & \\
\hline & & Content & & & & & \\
\hline & & Position & & & & Yes & No \\
\hline & & Volume of voice & & & & & \\
\hline & & Tone of voice & & & & & \\
\hline & & Facial Expression & & & & & \\
\hline & & Visual contact & & & & & \\
\hline & & Content & & & & & \\
\hline & & Position & & & & Yes & No \\
\hline & & Volume of voice & & & & & \\
\hline & & Tone of voice & & & & & \\
\hline & & Facial Expression & & & & & \\
\hline & & Visual contact & & & & & \\
\hline & & Content & & & & & \\
\hline
\end{tabular}

We looked for connections that could be created in the group: the role of the framework, social levels, construction of meaning. The framework of the group was very important for determining the links and social levels constituting the role of interactions between the different participants as well as between the therapists and participants. The construction within the group enabled the construction of a therapeutic process. We benefitted from the help of a peer-helper whose usefulness was demonstrated in autism spectrum disorders $[29,30]$. We focused group work on synergy and the notion of reinforcement through the experiences between participants.
Because of this, participants allowed themselves more easily to accept progress and we worked on self-reinforcement which was then amplified through peer feedback. Thanks to a sharing of skills, we were able to work on skill transfer, comparing representations and solidarity between participants. We actively worked on the concepts of self-confidence, identity, belonging and competence.

Finally, two decisive factors in the success of this group therapy were the motivation of the participants to get beyond their situation, as well as their capacity for understanding and conceptualization. 


\section{Results}

The results obtained show that during the baseline phase (T0), the questionnaires revealed anxiety disorders in every patient accompanied by serious physiological symptoms. As shown in Figure 2, thanks to our therapeutic intervention, the repeated measures ANOVA during the different phases (TO; T1 and $\mathrm{T} 2$ ) showed a significant reduction in the anxiety disorders (STAI) $F(2,29=32.736, p<.0001)$, the physiological symptoms of anxiety (anxiety scale) F(2,29=143.299, p<.0001) as well as an improvement in self-esteem (rosenberg scale) $F(2,29=$ 43.516, $\mathrm{p}<.0001)$.

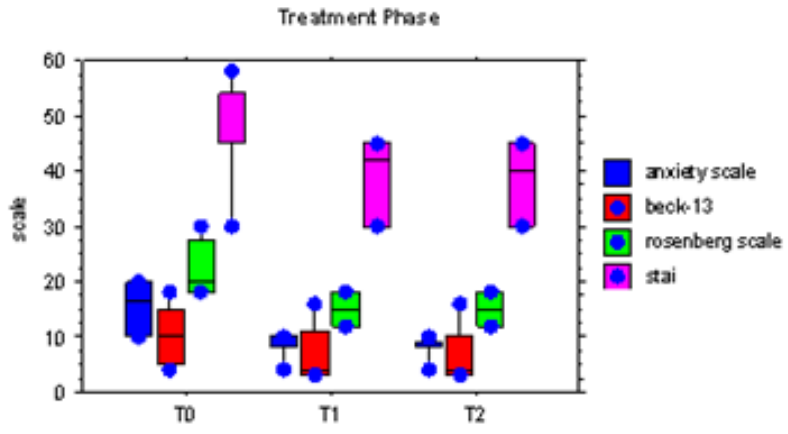

Figure 2 Therapeutic intervention during the different phases (T0; T1 and T2). The intervention showed a significant reduction in the anxiety disorders (STAI) F(2, $29=$ $32.736, p<.0001)$, the physiological symptoms of anxiety (anxiety scale) $F(2,29=143.299, p<.0001)$ as well as an improvement in self-esteem (rosenberg scale) $\mathrm{F}(2$, $29=43.516, p<.0001)$.

The evaluation conducted three months following the treatment ( $\mathrm{T} 2$ =follow-up phase) confirmed to us that the improvement reported at T1 was not a placebo effect and that it was maintained at T2. Thus, it was the stabilization of the results obtained between the intervention and the non intervention which enabled us to prove the effectiveness (established with the questionnaire) of the group therapy work $(t=19.795, d f=239, p<.0001)$.

The Spearman correlation of the 10 sessions conducted on participant fatigue before and after the group (rho=-3.356, $\mathrm{df}=39, \mathrm{p}=.0064$ ) and on the usefulness of the group (rho=.243, $\mathrm{df}=39, \mathrm{p}=.0041$ ) revealed significant differences. In terms of fatigue, we report that the participants were indeed more tired after the group session compared to before. This indicates that the group work requires their attention. For the usefulness of the group, over time the group session was perceived to be more and more useful and satisfying.

\section{Discussion}

The data we obtained in the group therapy are in line with the data gathered by $[5,19]$ which suggests that there is a deep engagement in the process of patient change. Although governments currently $[31,32]$ have guidelines that support care interventions directed at this population, the first research on $\mathrm{BCT}$ therapies with this population dates only from 2010 [19] and the research is still at a stage in which the descriptive process adds richness to our understanding of the group therapy option for this population.

A certain number of authors have described how BCT approaches can be adapted to the learning and interaction styles of ASD patients [18,33-35], but little focus has been brought to the BCT group dynamic such as the interdependence of personal and environmental influences as defined in the Learning via one's peers section by Grossen [28], regulating systems and any circular interactions. The participants especially appreciated the predictability of the intervention, the structure of the sessions with a daily program. They appreciated the repetitions and the reinforcing of skills. They liked the scientific aspect of the BCT based on concrete proofs and not on abstract ideas as well as the fact that the group was kept small (max. 6 people). The facilitators enabled the transfer of skills between peers, something which largely promoted an improvement in self-esteem. We would like to insist upon the fact that these adults live in a situation of precarious mental and social health, as we have underlined in our articles on the profile of Asperger adults in Frenchspeaking Switzerland [36,37]. On the other hand, our participants all received their diagnosis as adults, and because they have good intellectual capacities, they do not fall into the category of psychiatric patients because, firstly, the professionals are not trained for these patients, and secondly, Asperger's patients do not identify with patients suffering from other pathologies [19]. When an adult with ASD becomes familiar with his environment and what is expected of him, he can become sufficiently competent and confident so that this blindness is hidden. This is how certain adults with ASD remain undiagnosed and end up looking for help because of emotional difficulties (anxiety, anger, depression) linked to particular contexts such as new romantic relationships, inability to keep a job, money management, unsatisfied sexual needs. There is a lot of confusion in diagnostic criteria because certain symptoms described for ASD's are masked or not clear. More than that, a professional can interpret the signs of ASD's (distrust, explosivity, impulsivity, emotional lability) as symptoms of a borderline or psychotic personality disorder or the therapist tends to turn toward a diagnosis of depression, general anxiety disorders, phobias or obsessive-compulsive disorders when there are stereotypies and ritual behavior [38].

Our study shows that working on social skills does help patients reduce their anxiety and acquire self-control over their behaviors and it also appeared to improve their selfesteem.

The therapeutic alliance with the group participants, their availability and their engagement in the process of cognitive behavioral therapy work also made it possible to reduce their most significant anxiety and which was restricting their autonomy, their self-image and their self-control.

The psycho-educational approach also helped the participants understand the phenomena triggering their difficulty and the related consequences. 
Three major conclusions can be drawn from our study. Firstly, it shows that individuals living with Asperger's can improve their social skills in a group setting. Because of this project, the patients reduced their anxiety disorders. Secondly, it confirms that personnel can offer CBT interventions that are effective and adapted to this population provided that they are trained [39-44]. Finally, the use of a peer-helper enabled a deeper sharing of skills.

The care personnel is an unused resource which can be used to increase the availability of CBT interventions for this often overlooked population. Recognizing the mental health issues of this vulnerable population and increasing the number of psychiatrists specialized in this area are major challenges for the future $[19,45]$.

\section{Acknowledgements}

We are grateful for the participants who gave us their consent for this study as well as their trust, as well as everyone in our team who worked together in this therapeutic approach.

\section{References}

1. Baio J (2008) Prevalence of Autism Spectrum Disorders.

2. Prevention CFDC (2014) Prevalence of Autism Spectrum Disorder Among Children Aged 8 Years 2: 1-22.

3. Hadjikhani N (2014) Scientifically deconstructing some of the myths regarding autism. Swiss Archives of Neurology and Psychiatry 165: 272-276.

4. DSM-V (2013) Diagnostic and Statistical Manual of Mental Disorders (5th edn). American Psychiatric Association: Washington.

5. Langdon PE (2013) Asperger syndrome and anxiety disorders (PAsSA) treatment trial: a study protocol of a pilot, multicentre, single-blind, randomised crossover trial of group cognitive behavioral therapy. BMJ Open 3: 1-7.

6. Russell E, Sofronoff K (2005) Anxiety and social worries in children with Asperger syndrome. Aust N Z J Psychiatry 39: 633-638.

7. Szatmari P (1998) Genetics of autism: overview and new directions. J Autism Dev Disord 28: 351-368.

8. Tantam D (1988) Lifelong eccentricity and social isolation. I. Psychiatric, social, and forensic aspects. Br J Psychiatry 153: 777-782.

9. Tantam D (1988) Lifelong eccentricity and social isolation. II: Asperger's syndrome or schizoid personality disorder? $\mathrm{Br}$ Psychiatry 153: 783-791.

10. Tantam D (1988) Asperger's syndrome. J Child Psychol Psychiatry 29: 245-55.

11. Lugnegard T, Hallerback MU, Gillberg C (2011) Psychiatric comorbidity in young adults with a clinical diagnosis of Asperger syndrome. Res Dev Disabil 32: 1910-1917.

12. Gillott A, Standen PJ (2004) Anxiety and stress in adults with autism. J Intellect Disabil Res 48: 335.
13. Gillott A, Standen PJ (2007) Levels of anxiety and sources of stress in adults with autism. J Intellect Disabil 11: 359-370.

14. Gould RA, Buckminister S (1997) Cognitive-behavioural and pharmacological treatment for social phobia: a meta-analysis. Clin Psychol Sci Pract 4: 291-306.

15. Antony MM, Rowa K (2005) Evidence-based assessment of anxiety disorders in adults. Psychol Assess 17: 256-66.

16. Rowa K, Antony MM (2005) Psychological treatments for social phobia. Can J Psychiatry 50: 308-316.

17. Rowa K (2005) Why are some obsessions more upsetting than others? Behav Res Ther 43: 1453-1465.

18. Cardaciotto L, Herbert AD (2004) Cognitive behavior therapy for social anxiety disorder in the context of Asperger's syndrome: a single-subject report. Cogn Behav Pract 11: 75-81.

19. Weiss JA, Lunsky Y (2010) Group cognitive behaviour therapy for adults with Asperger syndrome and anxiety or mood disorder: a case series. Clin Psychol Psychother 17: 438-446.

20. Boisvert JM (1987) Le développement de la compétence sociale: un guide pratique Revue de modification du comportement 17 : 1.

21. Curran JP (1980) The socially inadequate patient: incidence rate, demographic and clinical features, and hospital and posthospital functioning. J Consult Clin Psychol 48: 375-382.

22. Kazdin AE (1979) Effects of covert modeling and coding of modeled stimuli on assertive behavior. Behav Res Ther 17: 53-61.

23. DSM-IV (2000) DSM-IV-TR : diagnostic and statistical manual of mental disorders (4th edn). American Psychiatric Association: Washington.

24. Heavens D, Murphy GH, Langdon PE (2012) People with Asperger syndrome and anxiety (PAsSA) trial treatment manual (Version 3.0). University of East Anglia, Norwich.

25. Cungi C (2001) La thérapie cognitive : manuel du thérapeute (2ed edn) Rumilly: Afforthecc.

26. Cungi C (2001) L'affirmation de soi : manuel du thérapeute. Rumilly: Afforthecc.

27. Cungi C (1996) Savoir s'affirmer. Retz ed, Paris.

28. Grossen M (1990) L'articulation entre l'individu et le groupe dans la construction de l'espace thérapeutique. in colloque "La construction de l'espace thérapeutique". Université de Neuchâtel.

29. Liebal K (2008) Helping and cooperation in children with autism. J Autism Dev Disord 38: 224-238.

30. Petursdottir AL (2007) The effects of scripted peer tutoring and programming common stimuli on social interactions of a student with autism spectrum disorder. J Appl Behav Anal 40: 353-357.

31. Excellence NIHC (2012) Autism: recognition, referral, diagnosis and management of adults on the autism spectrum, NICE Clinical Guideline 142. British Psychological Society \& The Royal College of Psychiatrists: London.

32. Santé HA (2012) Synthèse de la Recommandation de bonne pratique : Autisme et autres TED. France.

33. Anderson S, Morris J (2006) Cognitive behavior therapy for people with Asperger syndrome. Behavioral and Cognitive Psychotherapy 34: 293-303. 
34. Attwood T (2004) Cognitive behaviour therapy for children and adults with Asperger's syndrome. Behaviour Change 21: 147-161.

35. Gaus VL (2007) Cognitive-behavioral therapy for adult Asperger syndrome. New York: Guilford Press.

36. Giuliani F, El Korh P (2016) Adult with Autistic Spectrum Disorders: Specialized Treatment. Adv Tech Biol Med 4: 1.

37. Giuliani F, El Korh P (2016) Profils des patients suivis dans une unité spécialisée Asperger. Swiss Archives of Neurology and Psychiatry 167: 88-93.

38. Barnhill GP (2001) Social attributions and depression. Focus on Autism and other Developmental Disabilities 16: 46.

39. McGillivray JA, McCabe MP, Kershaw MM (2008) Depression in people with intellectual disability: an evaluation of a staffadministered treatment program. Res Dev Disabil 29: 524-536.

40. Willner P, Tomlinson S (2007) Generalization of anger-coping skills from day-service to residential settings. J Appl Res Intellect Disabil 20: 553-562.
41. Giuliani F, El Korh P (2014) Psychothérapie de personnes vivant avec le syndrome Asperger autour de la caetextia. Swiss Archives of Neurology and Psychiatry 165: 298-305.

42. Giuliani F, El Korh P (2015) Case study: the contribution of the neurosciences in the treatment of the anxiety disorders at two people living with autism spectrum disorders and intellectual disabilities. RFCCC 10: 8-22.

43. Giuliani F, El Korh P (2016) Is Storytelling Therapy Useful for Children with Autism Spectrum Disorders and Severe Mental Retardation? Advanced Techniques in Biology \& Medicine 4: 166-201.

44. Giuliani F, Perrenoud V, Favrod J (2014) Using eye-tracking as support for the TEACCH program and two teenagers with autism-spectrum disorders. Fran J Beh clinic 19: 39-56.

45. Grasset F (2008) Mental retardation and psychiatry. Rev Med Suisse 4: 1976-1978. 